\title{
Canaliculitis caused by Enterobacter cloacae: report of a case
}

\author{
L. C. CHUMBLEY
}

From the Olmsted Medical and Surgical Group, 210 SE Ninth Street, Rochester, Minnesota, USA

SUMmaRY A 65-year-old man developed canaliculitis first of the left lower and later of the left upper lacrimal canaliculus. Mucoid material expressed from the upper canaliculus grew a pure culture of Enterobacter cloacae, the colonies of which had a stringy, tenacious, mucoid property consistent with the clinical findings. Treatment with topical chloramphenicol and oral ampicillin was followed by complete resolution.

Canaliculitis is a problem infrequently seen by the ophthalmologist. The occurrence of canaliculitis due to actinomyces has been well described. ${ }^{2}$ I report here a case of canaliculitis due to Enterobacter cloacae, an organism which to my knowledge has not been described before as causing this infection. Furthermore, the microbiological characteristics of the organism explained some of the clinical features seen.

\section{Case report}

A 65-year-old farmer developed swelling and tenderness of the medial canthal portion of the left lower eyelid and epiphora. After a period of 2 months a large 'blob' of sticky, whitish material was discharged spontaneously into the conjunctival sac, and the symptoms were relieved. Within 3 weeks, however, he noted marked swelling and tenderness (but not epiphora) affecting the medial canthal portion of the left upper eyelid, and an accumulation of mucopurulent discharge. This persisted despite therapy. From the beginning of his original symptoms treatment had consisted of topical dexamethasone $0.1 \%$ and Cortisporin (polymyxin B, neomycin sulphate, hydrocortisone) ophthalmic drops, as frequently as 3 hourly, with systemic erythromycin $250 \mathrm{mg}$. 4 times daily given in courses of 4 weeks' duration 3 times. The problem had persisted for 7 months when the patient consulted me.

At the time of my first examination 7 months after onset of symptoms I noted marked swelling and Correspondence to Dr L. C. Chumbley, St John Ophthalmic Hospital, PO Box 19960, Jerusalem, Israel. thickening of the medial canthal region of the left upper eyelid with pouting of the left superior lacrimal punctum (Fig. 1). Irrigation through the left inferior lacrimal punctum produced prompt appearance of irrigation fluid in the nasopharynx. Because of tenderness no attempt was made to probe the superior lacrimal canaliculus. By means of counter pressure between 2 cotton tipped applicator sticks in the affected upper medial canthus region and slowly massaging from extreme medially towards the

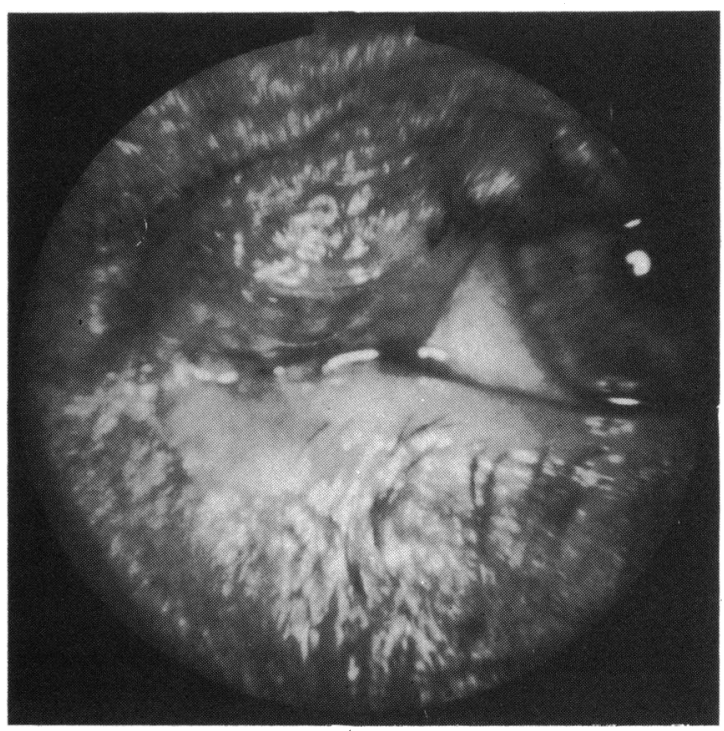

Fig. 1 Appearance of patient with left upper canaliculitis showing marked swelling of involved medial canthal region. 


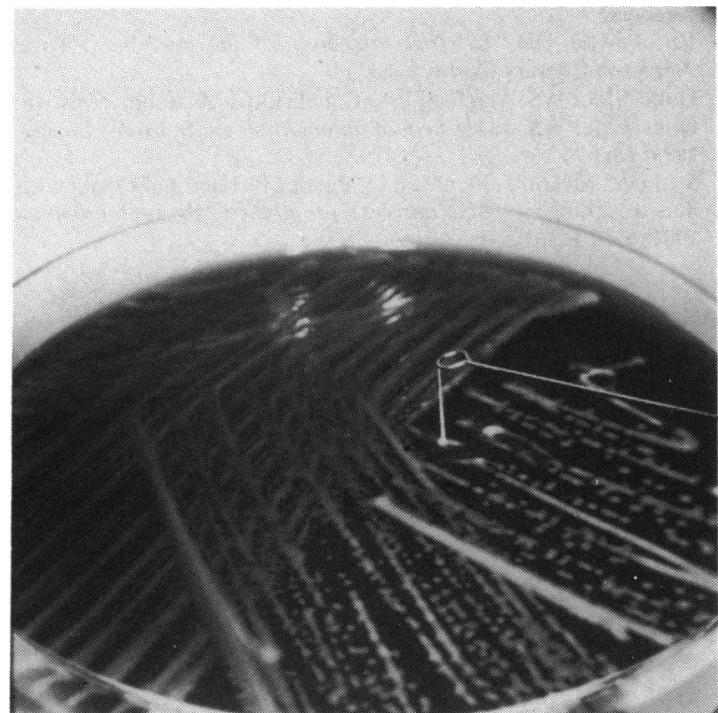

Fig. 2 Culture plate of pure Enterobacter cloacae isolated from material expressed from canaliculus. Note stringy, tenacious, mucoid stranding.

superior lacrimal punctum a large amount of whitish pale yellow material was expressed from the superior lacrimal canaliculus. This material was sticky and tenacious and resembled inspissated toothpaste being squeezed from a tube.

Culture of this material on blood agar grew a pure culture of an organism subsequently identified by special techniques as Enterobacter cloacae. Gram negative rods were identified in the initially expressed material. The cultural characteristics of the organism were striking, with a marked mucoid tenacity producing long mucoid strands when the colonies were manipulated with a bacteriological wire loop (Fig. 2). No actinomyces organisms were noted on Gram or silver stains of the material.

Using the laboratory results of antibiotic sensitivities as a guide to therapy I began treatment with hot compresses, topical chloramphenicol ophthalmic drops 3 times daily to the left eye, and ampicillin 250 mg 4 times daily by mouth for 3 weeks. The swelling and tenderness improved after this therapy was begun. When the medication ran out, however, the swelling and tenderness of the upper left medial canthal region became acutely worse. After 2 days of extreme pain the area of tenderness 'broke loose' with the expulsion of a large glob of mucoid material followed within several hours by the further expulsion of 2 small lumps of similar material. This resulted in decreased swelling and greater comfort. Treatment with topical chloramphenicol and oral ampicillin was resumed as before and continued for 4 weeks.

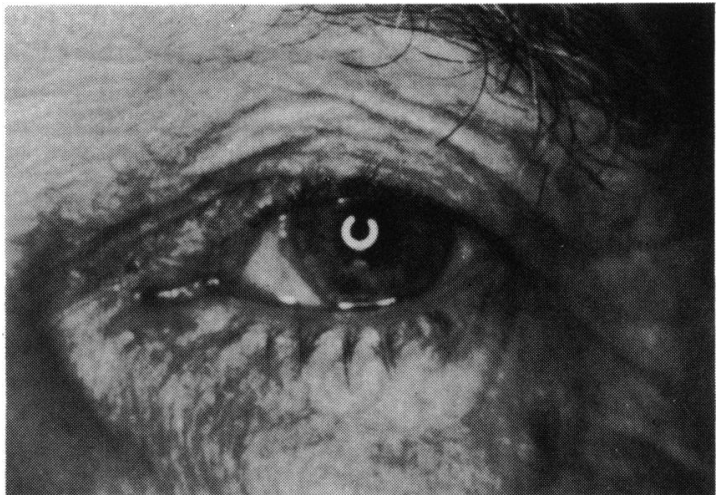

Fig. 3 Appearance of patient 2 months after starting therapy. Problem has been resolved.

Complete resolution of all symptoms and signs occurred (Fig. 3), and at a follow-up examination one year later the patient had remained disease free. Probing was easily performed at this follow-up visit, and no obstructions or concretions were encountered.

\section{Discussion}

Canaliculitis is a problem not often encountered in daily ophthalmic practice. The signs of swelling and tenderness in the region of the involved canaliculus together with pouting of the relevant lacrimal punctum will lead the clinician to suspect this diagnosis.

Canaliculitis is most commonly caused by Actinomyces israeli (streptothrix). ${ }^{\prime}$ This is a Gram-positive branching organism possessing conidia. It is a strict anaerobe and is best isolated with brain-heart infusion media in a completely oxygen free environment, such as a gas pack jar. The organism is recovered from anaerobic sites in the gingivae associated with dental caries. Canaliculitis due to Actinomyces israeli is characterised by swelling in the region of the involved canaliculus, yellowish discharge, and the production of concretions in the canaliculi. Treatment is with penicillin, and all concretions must be removed to effect a cure. ${ }^{3}$

Enterobacter cloacae as a cause of canaliculitis, described in this report, has not been described before to my knowledge. E. cloacae is a common intestinal organism found also in soil and water. It is a Gram-negative rod.

Enterobacter cloacae forms colonies with a tenacious mucoid physical property as was noted in this case. This characteristic microbiological property accounted for the clinical findings of tenaciously adherent mucoid material which was both expressed 
and spontaneously expelled. Treatment with appropriately chosen topical and systemic antibiotics was effective in producing a clinical cure. The inappropriate use of topical corticosteroids, continued over many months at the outset, may have played a role in producing the full blown establishment of canalicultitis which was observed clinically.
References

1 Fedukowicz HB. External infections of the eye. New York: Appleton Century Crofts, 1963: 140.

2 Duke-Elder WS, MacFaul PA. Canaliculitis. Actinomycosis. In: Duke-Elder WS. ed. System of ophthalmology, St Louis: Mosby, 1974: 13: 696.

3 Seal DV, McGill J, Flanagan D, Purrier B. Lacrimal canaliculitis due to Arachnia (Actinomyces) propionica. Br J Ophthalmol $1981 ; 65: 10-3$. 